\title{
Publisher Correction: High-resolution myogenic lineage mapping by single-cell mass cytometry
}

Ermelinda Porpiglia, Nikolay Samusik, Andrew Tri Van Ho, Benjamin D. Cosgrove, Thach Mai, Kara L. Davis, Astraea Jager, Garry P. Nolan, Sean C. Bendall, Wendy J. Fantl and Helen M. Blau

Correction to: Nature Cell Biology https://doi.org/10.1038/ncb3507, published online 17 April 2017.

In the version of this Article originally published, the name of author Andrew Tri Van Ho was coded wrongly, resulting in it being incorrect when exported to citation databases. This has been corrected, though no visible changes will be apparent.

Published online: 5 March 2018

https://doi.org/10.1038/s41556-018-0043-1

\section{Publisher Correction: Hitchhiking on selective autophagy}

Christian Münch and Ivan Dikic

Correction to: Nature Cell Biology https://doi.org/10.1038/s41556-018-0036-0, published online 25 January 2018.

In the version of this News \& Views originally published, owing to a technical error some incorrect characters were introduced into the second sentence of the main text, starting "Autophagy can be divided into non-selective...", where "caspringDE@2017n" should have read 'can'. This has now been corrected in all versions of this News \& Views.

Published online: 19 April 2018

https://doi.org/10.1038/s41556-018-0051-1

\section{Publisher Correction: MSK1 regulates luminal cell differentiation and metastatic dormancy in $\mathrm{ER}^{+}$breast cancer}

Sylwia Gawrzak, Lorenzo Rinaldi, Sara Gregorio, Enrique J. Arenas, Fernando Salvador, Jelena Urosevic, Cristina Figueras-Puig, Federico Rojo, Ivan del Barco Barrantes, Juan Miguel Cejalvo, Marta Palafox, Marc Guiu, Antonio Berenguer-Llergo, Aikaterini Symeonidi, Anna Bellmunt, Daniela Kalafatovic, Anna Arnal-Estapé, Esther Fernández, Barbara Müllauer, Rianne Groeneveld, Konstantin Slobodnyuk, Camille Stephan-Otto Attolini, Cristina Saura, Joaquín Arribas, Javier Cortes, Ana Rovira, Montse Muñoz, Ana Lluch, Violeta Serra, Joan Albanell, Aleix Prat, Angel R. Nebreda, Salvador Aznar Benitah and Roger R. Gomis

Correction to: Nature Cell Biology https://doi.org/10.1038/s41556-017-0021-z, published online 22 January 2018.

In the version of this Article originally published, the boxes framing the two plots in Fig. 1g were misaligned from the axes due to a technical error. This has now been corrected in all versions of the Article.

Published online: 19 April 2018

https://doi.org/10.1038/s41556-018-0052-0 